\title{
Dispatch Strategy as a Design Variable in Hybrid Renewable Energy System Optimisation
}

\author{
Mohammed Althani \\ School of Engineering \\ University of Aberdeen \\ Aberdeen, UK \\ m.althani.19@abdn.ac.uk
}

\author{
Alireza Maheri \\ School of Engineering \\ Centre for Energy Transition \\ University of Aberdeen \\ Aberdeen, UK \\ alireza.maheri@abdn.ac.uk
}

\begin{abstract}
In hybrid renewable energy system (HRES) with multiple storage and auxiliary/backup components different dispatch strategies can be defined based on the usage and charging order of these components. This paper presents a new formulation for size and configuration optimisation of HRES by including the dispatch strategy as a design variable. The new formulation is implemented in the optimisation tool MOHRES and is used to conduct three case studies with various scenarios. It is shown that compared to traditional method of predefining the dispatch strategy prior to size optimisation, inclusion of the dispatch strategy in size optimisation leads to superior solutions.
\end{abstract}

Keywords- optimisation, hybrid renewable energy systems, wind-PV-battery-diesel-fuel cell, dispatch strategy, MOHRES.

\section{INTRODUCTION}

Renewable energy plays a vital role in the global energy transition plans and power sector decarbonisation goals. Abundance, widely available, sustainable, and emissions free are the main features of renewable energy sources. However, their intermittent nature impacts the reliability of renewable energy systems and significantly if based on a single renewable energy source.

Hybrid renewable energy systems (HRES) are implemented to overcome the challenge imposed by the energy fluctuation where more than one primary energy source, with at least one renewable energy source, are used (e.g. wind-diesel system) [1]. Consequently, HRES have large penetration in power generation due to their capability to meet the electricity demand in a reliable and relatively more environmentally friendly way for both grid-connected and standalone applications [2-4].

Energy storage systems (ESS) are another contributor to reliability enhancement when added to renewable energy systems. Availability of different auxiliary and storage components with finite resources of a desired system makes trade-off between cost, reliability, and environmental impact inevitable hence the necessity of size and configuration optimisation of HRES.

Flow of energy within the system is another important matter in HRES to serve the demand load continuously and to keep the system balanced. A dispatch strategy is needed for the energy management system (EMS) to specify the order of charging ESS during energy surplus period when more than one ESS is available. Similarly, discharging order is needed to supplement power shortage during power deficit period.
The common practice is to set the dispatch model prior to size optimisation process or test different dispatch strategies to obtain best possible output of an optimal size of HRES [58]. For instance, PV-Diesel-Battery and Wind-PV-BatteryDiesel of HRES configurations presented in [9] and [10] respectively, battery discharging is given the priority to meet the demand level when primary energy supply falls short. In [11] only three out of four dispatch strategies are predefined and tested for PV-Battery- Fuel Cell hybrid system. on the other hand, all possible dispatch models derived from WindPV-Diesel-Battery-Fuel Cell/Electrolyser hybrid system configuration has been tested and the sensitivity of dispatch strategy on HRES optimisation is reported in [12].

In this paper inclusion of the dispatch strategy in the optimisation process as a design variable is investigated using the proposed formulation. Scenario-based case studies are conducted to obtain candidate solutions for single objective optimisation using genetic algorithm.

\section{INCLUSION OF DISPATCH STRATEGY IN THE SIZE OPTIMISATION FORMULATION OF HRES}

With the inclusion of the dispatch model in the optimisation process the dispatch strategy of auxiliary and storage components is not defined by the designer, it is obtained by the optimisation instead along with the components size and configuration of the system. The formulation and simulation of the system optimisation process are explained in the following subsections.

\section{A. Problem Formulation of Optimisation}

This subsection illustrates the new formulation using the design variables and qualities. The design variables can be divided into two categories: size variables $\vec{X}_{S}$ and dispatch model variables $\vec{X}_{d}$. Each individual design candidate is represented by a vector $\vec{X}$ where:

$$
\begin{gathered}
\vec{X}=\vec{X}_{s} \cup \vec{X}_{d} \\
\vec{X}_{s}=\left\{s_{1}, s_{2}, s_{3}, \ldots, s_{N}\right\} \\
\vec{X}_{d}=\left\{\vec{O}_{u}, \vec{O}_{c}\right\} \\
\vec{O}_{u} \subseteq\left\{u_{1}, u_{2}, u_{3}, \ldots, u_{n}\right\} \\
\vec{O}_{c} \subseteq\left\{c_{1}, c_{2}, c_{3}, \ldots, c_{m}\right\}
\end{gathered}
$$


where, $s$ is the size of the system's component, $\vec{O}_{u}$ is usage order of auxiliary and ESS components, $\vec{O}_{c}$ charging order of storage components, $N$ is the number of components of the system, $n$ is the number of auxiliary power supply components, and $m$ is the number of energy storage components.

A single objective optimisation can be expressed by the following:

$$
\begin{gathered}
\min / \max Y_{j} ; Y_{j} \in \vec{Y} \\
\text { s.t. } \\
\vec{Y}_{a} \leq \vec{Y}_{i} \leq \vec{Y}_{b} ; \vec{Y}_{i} \subseteq \vec{Y}-\left\{Y_{j}\right\} \\
\vec{X}_{s}^{l} \leq \vec{X}_{s} \leq \vec{X}_{s}^{u} \\
\vec{X}_{d}=\left\{\vec{O}_{u} \cup \vec{O}_{c} \mid \vec{O}_{u} \subseteq U^{n ! \times n}, \vec{O}_{c} \subseteq C^{m ! \times m}\right\}
\end{gathered}
$$

where, $Y_{j}$ is a desired objective of optimisation subjected to some constraints $\vec{Y}_{a}$ and $\vec{Y}_{b}$ with lower and upper limits of system components size $\vec{X}_{s}^{l}$ and $\vec{X}_{s}^{u}$ respectively, and $u$ and $c$ represent the discrete search domains of usage and charging orders respectively.

\section{B. Simulation Methodology}

The flow of energy is controlled by EMS (Fig. 1), adapting the dispatch model of auxiliary and storage components in the system, is explained in this subsection. The simulation is carried out on hourly average basses. The summation of renewable power produced by primary renewable energy source is compared with demand load level. When the renewable power is more than demand load, charging order, which is generated by optimisation process, is executed till maximum state of the first storage component is reached. Then the next component ranked by $\vec{O}_{c}$ one component at a time. In the deficit power situation, usage order is implemented. Storage and auxiliary components supply the power to meet the demand load consistent with $\vec{O}_{u}$. However, when the auxiliary component (e.g. diesel generator) is in operation in its nominal power and exceed the demand load, the excess power will charge the storage component following the order of $\vec{O}_{c}$.

\section{CASE STUDIES}

In this section, various scenarios of three case studies are presented and summarised in TABLE I, TABLE II, and TABLE III. The optimisation is conducted by the optimisation tool MOHRES (Multi-objective Optimisation of Hybrid Renewable Energy System under uncertainties). The new formulation proposed in this study has been implemented in MOHRES where (2), (4), and (5) can be expressed as the following:

$$
\begin{aligned}
& \vec{X}_{S}=\left\{R_{W T}, A_{P V},\right.\left.n_{B}, P_{D, \text { nom }}, P_{f c, \text { nom }}, P_{\text {elec }, \text { nom }}\right\} \\
& \vec{O}_{u} \subseteq\{B, F C, D\} \\
& \vec{O}_{c} \subseteq\{B, F C\}
\end{aligned}
$$

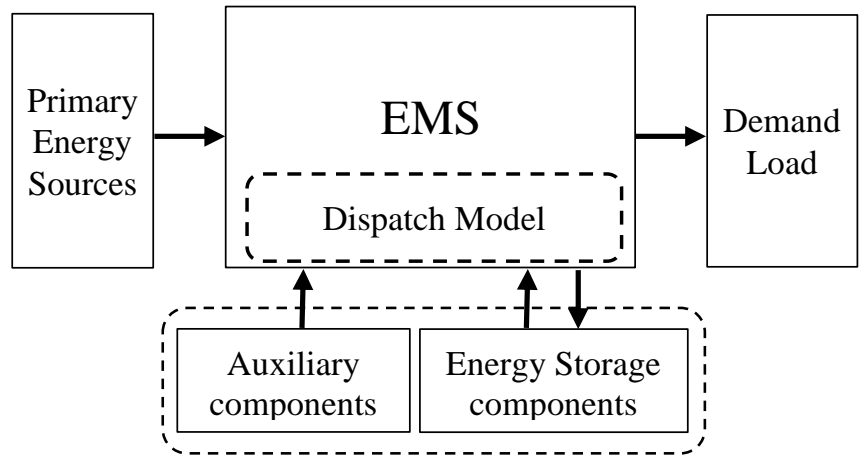

Fig. 1. Energy flow of hybrid renewable energy system.

Since a genetic algorithm is used, the size and dispatch model strings are represented by real and integer numbers respectively. The first six elements of $\vec{X}$ specifies the size of each component in the system as follows: $R_{W T}$ is the wind turbine rotor radius, $A_{P V}$ is the total PV panels area, $n_{B}$ is the number of unit battery, and $P_{D, n o m}, P_{f c, n o m}$, and $P_{\text {elec,nom }}$ are nominal power of diesel generator, fuel cell, and electrolyser, respectively. The power output of each component in the system is calculated by the mathematical models presented in $[3,4,13,14]$.

Unlike the size variables, $\vec{O}_{c}$ and $\vec{O}_{u}$ are quantified by their elements rank. In other words, their variability is in their elements ranks, which is randomly created in initial population, passed to the new offspring from parents in crossover, and regenerated in mutation. The combination of these two strings forms the dispatch model for EMS. The length of $\vec{O}_{c}$ and $\vec{O}_{u}$ depend on the available auxiliary and storage components where B, FC, and D stand for Battery, Fuel Cell, and Diesel, respectively. Therefore, the remaining elements of $\vec{X}$ after the sixth element is the dispatch model formed by usage and charging orders.

The design qualities vector $\vec{Y}$ is evaluated by economic $\left(\vec{Y}_{1}\right)$, technical $\left(\vec{Y}_{2}\right)$, and environmental $\left(\vec{Y}_{3}\right)$ measures using the mathematical models proposed in $[2,3,13,15,16]$ :

$$
\begin{gathered}
\vec{Y}_{1}(\vec{X})=\{T L S C, L C E\} \\
\vec{Y}_{2}(\vec{X})=\left\{U_{t}, B O_{t}, B O_{a v}, B O_{\text {max }}, M T B F, P_{\text {excess }}\right\} \\
\vec{Y}_{3}(\vec{X})=\left\{C O_{2}, p\right\} \\
\vec{Y}=\vec{Y}_{1} \cup \vec{Y}_{2} \cup \vec{Y}_{3}
\end{gathered}
$$

where, TLSC, $L C E$, and MTBF stand for total lifespan cost, levelised cost of energy and mean time between failures respectively; $U_{t}$ is total unmet load, $P_{\text {excess }}$ is net excess power, $\mathrm{CO}_{2}$ is carbon dioxide emissions, $p$ is renewable energy penetration; and $B O_{t}, B O_{a v}$, and $B O_{\text {max }}$ respectively are total, average, and maximum power blackout durations.

\section{A. Case Study 1: Expanding an Existing System to Meet Future Demand}

This case study is designed for expanding an existing Wind-FC system $\vec{X}_{S}=\{11.6,0,0,0,8400,5900\}$ serving the current demand load provided in Fig. 2. The renewable resources of the site are assumed to be equal to the values shown in Fig. 3 and Fig. 4. 
The first scenario is that a HRES is required to meet double the current demand load with a minimum levelised cost of energy. Two optimisations have been performed. The first optimisation (CS1.1) is conducted for the optimum size of the system where minimum size of wind turbine, fuel cell, and electrolyser is the existing system size. The dispatch model is fixed (i.e. the dispatch model is predefined prior to the optimisation). The second optimisation (CS1.2) is similar to

CS1.1 excluding the dispatch model which is not defined but included in the optimisation as a design variable. Both candidate systems are shown in TABLE IV.

\section{B. Case Study 2: Low Wind Energy}

In this case study it is assumed that the wind energy available for CS1.2 is $30 \%$ less due to the increase of the length of surface roughness. Consequently, the performance of the HRES obtained by the optimisation of CS1.2 is changed. Assessment of the system is carried out considering the wind energy reduction (CS2.1). Then an optimisation is conducted to find the optimum dispatch model (CS2.2). The results are presented in TABLE $\mathrm{V}$.

\section{Case Study 3: Integration of Two Existing Distinct Standalone Systems}

A wind-Diesel system of size $\vec{X}_{s}=\{7.3,0,0,13600,0,0\}$ and a PV-Battery system of size $\vec{X}_{s}=\{0,294,208,0,0,0\}$ are two distinct standalone systems which operate in the same area with renewable resources provided in Fig. 3 and Fig. 4. Each system is serving its own demand load, both equal to the demand load shown in Fig. 2.

In PV-Battery system $\vec{O}_{u}$ and $\vec{O}_{c}$ are constant $\left(\vec{O}_{u}=\vec{O}_{c}=\right.$ $B$ ) since only battery is available as ESS while in Wind-Diesel $\vec{O}_{u}=D$ and $\vec{O}_{c}=\{\}$ because there are no storage components in the system.

However, optimising the dispatch model of the system is still valid since the optimisation is conducted for the two systems integration and using the full configuration (windPV-battery-diesel-fuel cell/electrolyser).

In the first optimisation problem formulation (CS3.1), we are looking for the optimum dispatch strategy which meets the total demand load with minimum LCE.

In the second optimisation problem formulation (CS3.2), again we are looking for the optimum dispatch strategy which meets the total demand load with minimum LCE. However, we assume that the size of PV panel and battery bank can be also reduced to lower the cost. Hence, the lower limits for the PV panel area and the number of battery units in the battery bank are zero while, the upper limits are equal to their currently existing size.

Assuming that the excess power of the integrated systems can be fed into a grid or utilised for other applications, in the third optimisation case (CS3.3), we aim at maximising excess or feed in power.

The fourth optimisation problem (CS3.4) is formulated similar to the third one but with an extra constraint on $\mathrm{CO}_{2}$ emission as an environmental restriction. The optimised integrated system and its performance are shown in TABLE VI.
TABLE I. OPTIMISATION FORMULATION SUMMARY OF CASE STUDY 1

\begin{tabular}{|l|l|l|}
\hline \multicolumn{2}{|c|}{ Case Study 1 } \\
\hline \multicolumn{2}{|c|}{ Opt. problem } & \multicolumn{1}{c|}{$\overrightarrow{\boldsymbol{X}}$} \\
\hline \multirow{3}{*}{ CS1.1 } & $\min L C E$ & $\vec{X}_{s}^{l}=\{11.6,0,0,0,8400,5900\}$ \\
& s.t. & $\vec{X}_{s}^{u}=\left\{R_{W T}^{u}, A_{P V}^{u}, n_{B}^{u}, P_{D, n o m}^{u}, P_{F C, n o m}^{u}, P_{E L, n o m}^{u}\right\}$ \\
& $U_{t}=0$ & $\vec{O}_{u}=\{B, F C, D\} a, \vec{O}_{c}=\{B, F C\}$ (fixed) \\
\hline \multirow{4}{*}{ CS1.2 } & $\min L C E$ & $\vec{X}_{s}^{l}=\{11.6,0,0,0,8400,5900\}$ \\
& s.t. & $\vec{X}_{s}^{u}=\left\{R_{W T}^{u}, A_{P V}^{u}, n_{B}^{u}, P_{D, n o m}^{u}, P_{F C, n o m}^{u}, P_{E L, n o m}^{u}\right\}$ \\
& $U_{t}=0$ & $\vec{O}_{u}, \vec{O}_{c}$ are design variables \\
\hline
\end{tabular}

TABLE II. OPTIMISATION FORMULATION SUMMARY OF CASE STUDY 2

\begin{tabular}{|l|c|l|}
\hline \multicolumn{2}{|c|}{ Case Study 2 } \\
\hline \multicolumn{2}{|c|}{ Opt. problem } & \\
\hline $\mathrm{CS} 2.1$ & - & $\vec{X}$ \\
\hline $\mathrm{CS} 2.2$ & $\min U_{t}$ & $\begin{array}{l}\vec{X}_{s}^{l}=\vec{X}_{s}^{u}=\{11.6,20,222,900,8400,5900\} \\
\vec{O}_{u}=\{B, D, F C\}, \vec{O}_{c}=\{F C, B\} \text { (fixed) }\end{array}$ \\
\hline & $\begin{array}{l}\vec{O}_{u}^{l}=\vec{X}_{s}^{u}=\{11.6,20,222,900,8400,5900\} \\
\vec{O}_{c} \text { are design variables }\end{array}$ \\
\hline
\end{tabular}

TABLE III. OPTIMISATION FORMULATION SUMMARY OF CASE STUDY 3

\begin{tabular}{|c|c|c|}
\hline \multicolumn{3}{|r|}{ Case Study 3} \\
\hline \multicolumn{2}{|c|}{ Opt. problem } & $\vec{X}$ \\
\hline CS3.1 & $\begin{array}{l}\min L C E \\
\text { s.t. } \\
\quad U_{t}=0\end{array}$ & $\begin{array}{l}\vec{X}_{s}^{l}=\vec{X}_{s}^{u}=\{7.3,294,208,13600,0,0\} \\
\vec{O}_{u}, \vec{O}_{c} \text { are design variables }\end{array}$ \\
\hline CS3.2 & $\begin{array}{l}\min L C E \\
\text { s.t. } \\
\quad U_{t}=0\end{array}$ & $\begin{array}{l}\vec{X}_{s}^{l}=\{7.3,0,0,13600,0,0\} \\
\vec{X}_{s}^{u}=\left\{7.3,294,208,13600, P_{F C, n o m}^{u}, P_{E L, n o m}^{u}\right\} \\
\vec{O}_{u}, \vec{O}_{c} \text { are design variables }\end{array}$ \\
\hline CS3.3 & $\begin{array}{l}\max P_{\text {excess }} \\
\text { s.t. } \\
\quad U_{t}=0\end{array}$ & $\begin{array}{l}\vec{X}_{s}^{l}=\{7.3,0,0,13600,0,0\} \\
\vec{X}_{s}^{u}=\left\{7.3,294,208,13600, P_{F C, n o m}^{u}, P_{E L, n o m}^{u}\right\} \\
\vec{O}_{u}, \vec{O}_{c} \text { are design variables }\end{array}$ \\
\hline CS 3.4 & $\begin{array}{l}\max P_{\text {excess }} \\
\text { s.t. } \\
\quad U_{t}=0 \\
\mathrm{CO}_{2} \\
\leq 8770\end{array}$ & $\begin{array}{l}\vec{X}_{s}^{l}=\{7 \cdot 3,0,0,13600,0,0\} \\
\vec{X}_{s}^{u}=\left\{7 \cdot 3,294,208,13600, P_{F C, n o m}^{u}, P_{E L, n o m}^{u}\right\} \\
\vec{O}_{u}, \vec{O}_{c} \text { are design variables }\end{array}$ \\
\hline
\end{tabular}

\section{RESULTS AND DISCUSSION}

TABLE III lists the optimum solutions for the first and second optimisation of HRES for Case Study 1. The first one is with the size generated by the optimisation process while the dispatch model (i.e. usage and charging orders) is specified manually. For the deficit power situation, battery is used first to supply the power shortage with respect to demand load. When the energy stored in the battery is insufficient, fuel cell operates by consuming the hydrogen stored in the hydrogen tank. If the fuel cell is operating at its nominal power and the power production is still fell short, the diesel generator is dispatched with its nominal power. Any excess power from the system is firstly used to charge battery then the hydrogen tank. Any excess power left after wards is dumped.

The HRES of CS1.1 is capable to meet the demand load with levelised cost of 44.6 cents per kWh. However, once we include the dispatch strategy in the optimisation problem as in CS1.2, we obtain a better solution. Inclusion of dispatch model impacted the size of PV panel, number of battery units, and diesel generator thus found an optimum solution with lower LCE of 43.1 cents per $\mathrm{kWh}$.

As a matter of fact, the first solution presented in TABLE $\mathrm{V}$ (CS2.1) shows the performance of CS1.2 system utilising 
lower wind energy resources. Clearly, the total unmet load using the size of HRES obtained in CS1.2 is expected to be greater than zero. So, the objective of CS2.2 is to minimise the total unmet load. The HRES of CS2.2, with optimum dispatch model, generates $1658 \mathrm{~kW}$ more than the previous one (CS2.1).

In Case Study 3, the integrated system with the optimised dispatch model (CS3.1) is generating electricity at LCE of 35.2 cents per $\mathrm{kWh}$. The second HRES (CS3.2) has a lower LCE than the first one. This is expected since PV panels and battery units are reduced. Noticeably, the optimum dispatch model for the first system (CS3.1) is an optimum one for the second HRES (CS3.2). Nevertheless, different dispatch model has been obtained for CS3.3. Although LCE of CS3.3 is the highest due to more operation hours of the diesel generator, the goal of the optimisation in this case was to maximise the net power excess which can be achieved by the existing components of HRES. The fourth scenario of case study 3 (CS3.4) has environmental constraint which limits the diesel generator operation. Consequently, it generates less net power excess than CS3.3 and its optimum dispatch model is changed.

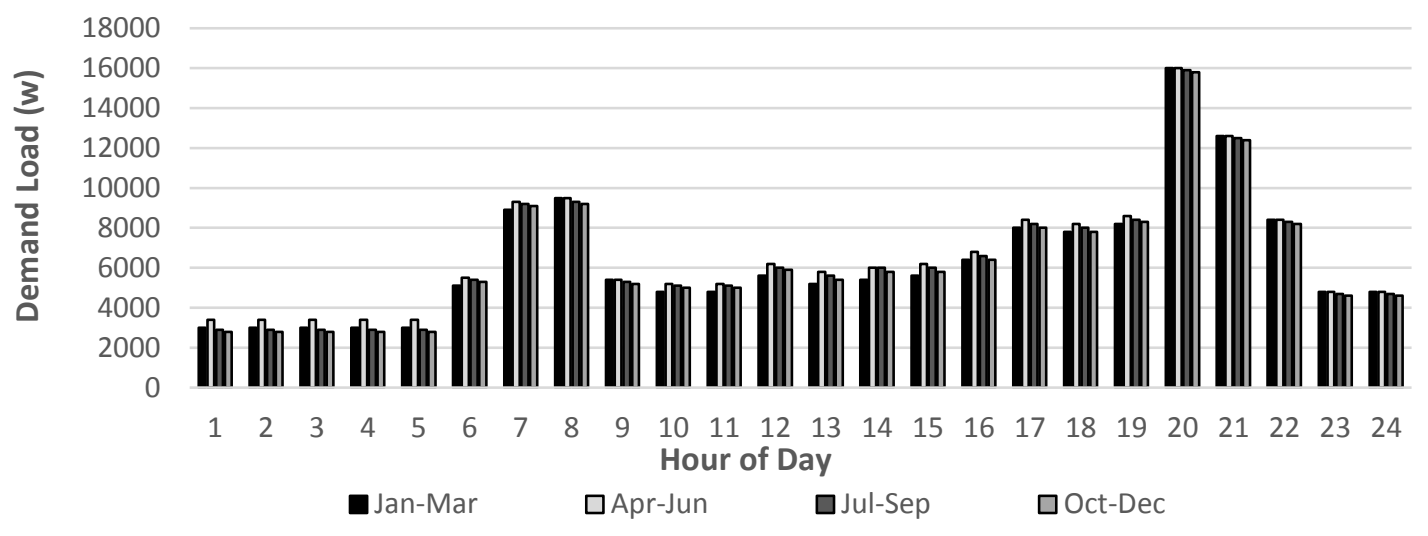

Fig. 2. Hourly averaged demand load of typical seasonal days.

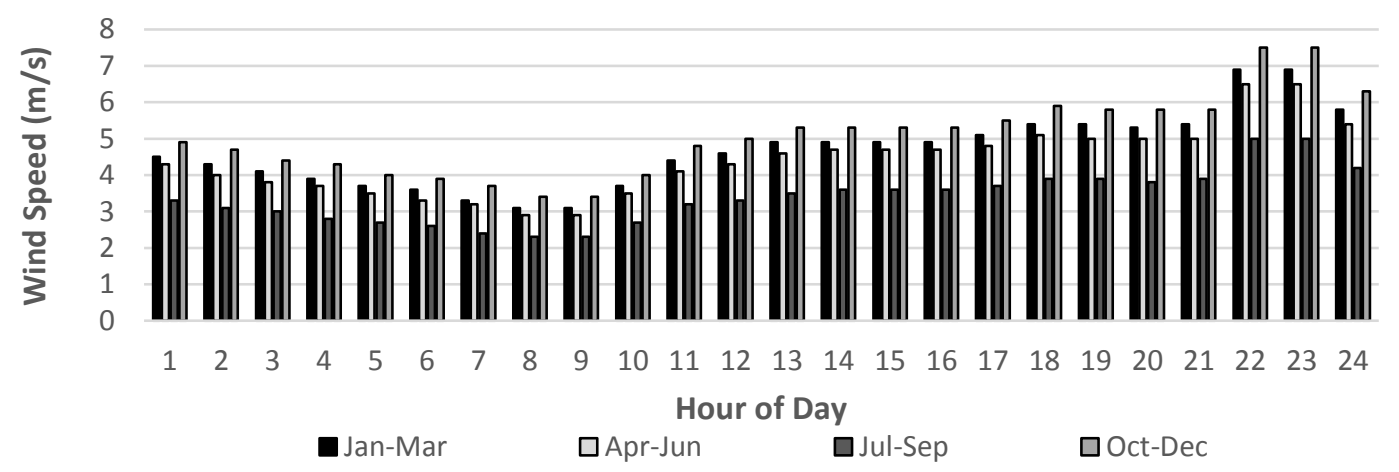

Fig. 3. Hourly averaged wind speed at reference height ( $h r e f=3 m)$ of typical seasonal days.

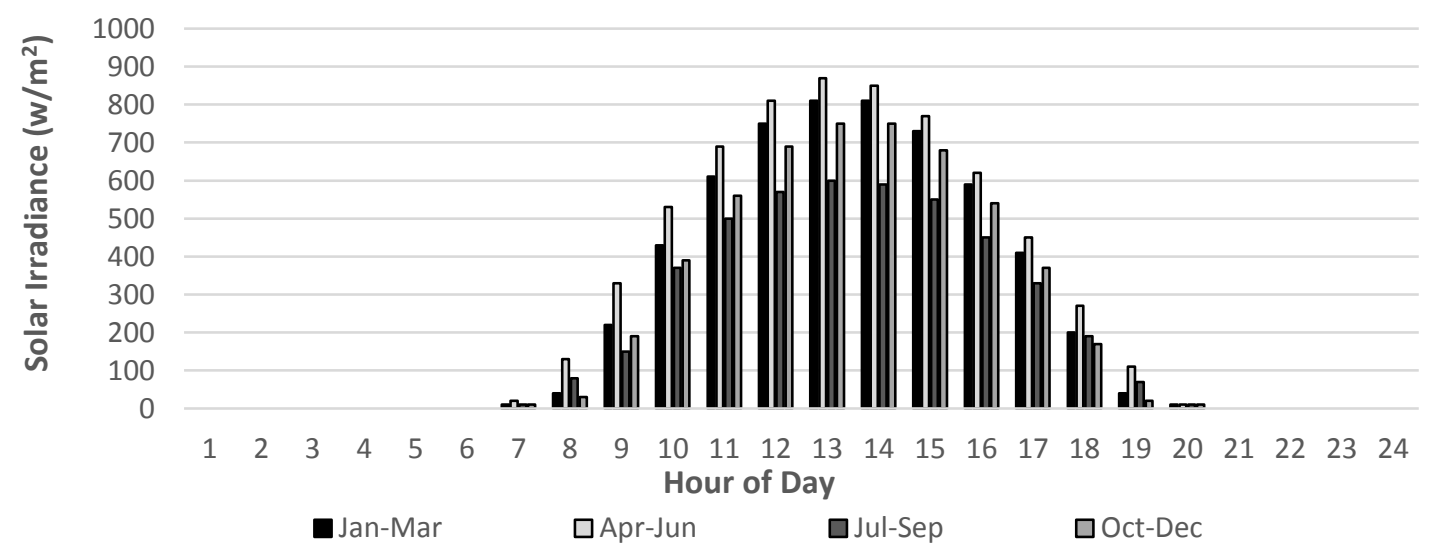

Fig. 4. Hourly averaged solar irradiance of typical seasonal days. 
TABLE IV. OPTIMISATION RESULTS FOR CASE STUDY 1

\begin{tabular}{|c|c|c|c|c|c|c|c|c|c|c|}
\hline $\begin{array}{c}\text { Case } \\
\text { Study }\end{array}$ & $\begin{array}{l}\boldsymbol{R}_{W T} \\
\left(\boldsymbol{m}^{2}\right)\end{array}$ & $\begin{array}{l}A_{P V} \\
\left(\boldsymbol{m}^{2}\right)\end{array}$ & $n_{B}$ & $\begin{array}{c}\boldsymbol{P}_{D, \text { nom }} \\
(\boldsymbol{W})\end{array}$ & $\begin{array}{c}\boldsymbol{P}_{F C, \text { nom }} \\
(\boldsymbol{W})\end{array}$ & $\begin{array}{c}\boldsymbol{P}_{E L, \text { nom }} \\
(\boldsymbol{W})\end{array}$ & $\overrightarrow{\boldsymbol{O}}_{\boldsymbol{u}}$ & $\overrightarrow{\boldsymbol{O}}_{c}$ & $\begin{array}{c}\text { LCE } \\
(\mathbb{C} / \boldsymbol{k W h})\end{array}$ & $\begin{array}{c}\boldsymbol{U}_{\boldsymbol{t}} \\
(\boldsymbol{k} W)\end{array}$ \\
\hline CS1.1 & 11.6 & 47 & 78 & 6900 & 8400 & 5900 & B-FC-D & B-FC & 44.6 & 0 \\
\hline CS1.2 & 11.6 & 20 & 222 & 900 & 8400 & 5900 & B-D-FC & FC-B & 43.1 & 0 \\
\hline
\end{tabular}

TABLE V. OPTIMISATION RESULTS FOR CASE STUDY 2.

\begin{tabular}{|c|c|c|c|c|c|c|c|c|c|c|}
\hline Case Study & $\overrightarrow{\boldsymbol{O}}_{u}$ & $\overrightarrow{\boldsymbol{O}}_{c}$ & $T L S C(\$)$ & $\begin{array}{c}\boldsymbol{L C E} \\
(\mathbb{C} / \boldsymbol{k W h})\end{array}$ & $B O_{t}(h)$ & $B \boldsymbol{O}_{a v},(\boldsymbol{h})$ & $B \boldsymbol{O}_{\max }(\boldsymbol{h})$ & $\begin{array}{c}\boldsymbol{U}_{\boldsymbol{t}} \\
(\boldsymbol{k W})\end{array}$ & $M T B F(h)$ & $\mathrm{CO}_{2}(\mathrm{~kg})$ \\
\hline CS2.1 & B-D-FC & FC-B & 823000 & 76.1 & 3935 & 14 & 23 & 33434 & 18 & 3760 \\
\hline CS2.2 & D-B-FC & B-FC & 899000 & 81.3 & 3559 & 10 & 17 & 31776 & 14 & 5260 \\
\hline
\end{tabular}

TABLE VI. OPTIMISATION RESULTS FOR CASE STUDY 3.

\begin{tabular}{|c|l|l|l|l|l|l|l|l|l|l|l|}
\hline $\begin{array}{c}\text { Case } \\
\text { Study }\end{array}$ & \multicolumn{1}{|c|}{$\begin{array}{c}\boldsymbol{R}_{\boldsymbol{W T}} \\
\left(\boldsymbol{m}^{2}\right)\end{array}$} & $\begin{array}{c}\boldsymbol{A}_{\boldsymbol{P V}} \\
\left(\boldsymbol{m}^{2}\right)\end{array}$ & \multicolumn{1}{|c|}{$\boldsymbol{n}_{\boldsymbol{B}}$} & $\begin{array}{c}\boldsymbol{P}_{\boldsymbol{D}, \text { nom }} \\
(\boldsymbol{W})\end{array}$ & $\overrightarrow{\boldsymbol{O}}_{\boldsymbol{u}}$ & $\overrightarrow{\boldsymbol{O}}_{\boldsymbol{c}}$ & $\begin{array}{c}\boldsymbol{L C E} \\
(\mathbb{C} / \boldsymbol{k W h})\end{array}$ & $\begin{array}{c}\boldsymbol{U}_{\boldsymbol{t}} \\
(\boldsymbol{k W})\end{array}$ & $\begin{array}{c}\boldsymbol{p} \\
(\%)\end{array}$ & $\begin{array}{c}\boldsymbol{P}_{\text {excess }} \\
(\boldsymbol{k W})\end{array}$ \\
\hline CS3.1 & 7.3 & 294 & 208 & 13600 & B-D & B & 35.2 & 0 & 135 \\
\hline $\mathrm{CS} 3.2$ & 7.3 & 163 & 128 & 13600 & B-D & B & 31.3 & 0 & 102 \\
\hline CS3.3 & 7.3 & 294 & 46 & 13600 & D-B & B & 41.0 & 0 & 8790 \\
\hline CS3.4 & 7.3 & 294 & 108 & 13600 & B-D & B & 34.7 & 0 & 135 \\
\hline
\end{tabular}

The optimisation results for CS1.1 and CS1.2 shown in TABLE IV proves that the inclusion of dispatch model in the optimisation as a design variable gives a superior solution with respect to the desired optimisation objective. The system performance of CS2.1 and optimisation result of CS2.2 in TABLE V shows that the optimum dispatch model for CS1.2 is not a global one although the optimum size of the system is. In other words, dispatch variables are as sensitive as size variables. The results of the third case study CS3.4 show that the dispatch model is sensitive to the constraints as well.

\section{CONCLUSION}

New formulation to include the dispatch model in the optimisation process as a design variable is presented. WindPV-Diesel-Battery-Fuel Cell/Electrolyser hybrid system configuration is used for size and dispatch model optimisation. Three scenario-based case studies are conducted using the new formulation. Superior solutions compared to predefined dispatch strategy has been obtained for single objective optimisation. Further investigation of the practicality and effectiveness of the dispatch model inclusion in the optimisation in terms of ESS performance degradation of frequent and irregular charging/discharging cycle is required to be conducted. Also, assessment of the level of EMS complexity imposed by the optimum dispatch model for the HRES should be considered. In the simulation of the system performance, more energy flow possibilities such as fuel cell and battery interaction, and charging based on a charging ratio can be introduced.

\section{REFERENCES}

[1] L. Olatomiwa, S. Mekhilef, M.S. Ismail and M. Moghavvemi, "Energy management strategies in hybrid renewable energy systems: A review," Renewable and Sustainable Energy Reviews, vol. 62, pp. 821-835, 2016.

[2] S. Leva and D. Zaninelli, "Hybrid renewable energy-fuel cell system: Design and performance evaluation," Electr.Power Syst.Res., vol. 79, pp. 316-324, 2009.

[3] C. Li, X. Zhu, G. Cao, S. Sui and M. Hu, "Dynamic modeling and sizing optimization of stand-alone photovoltaic power systems using hybrid energy storage technology," Renewable Energy, vol. 34, pp. 815-826, 2009.
[4] A. Maheri, "Multi-objective design optimisation of standalone hybrid wind-PV-diesel systems under uncertainties," Renewable Energy, vol. 66, pp. 650-661, 2014.

[5] Y. Kalinci, A. Hepbasli and I. Dincer, "Techno-economic analysis of a stand-alone hybrid renewable energy system with hydrogen production and storage options," Int J Hydrogen Energy, vol. 40, pp. 7652-7664, 2015.

[6] K. Chang, "A decision support system for planning and coordination of hybrid renewable energy systems," Decis.Support Syst., vol. 64, pp. 4-13, 2014.

[7] Z. Jing, J. Zhu and R. Hu, "Sizing optimization for island microgrid with pumped storage system considering demand response," Journal of Modern Power Systems and Clean Energy, vol. 6, pp. 791-801, 2018.

[8] F. Vivas, A. De las Heras, F. Segura and J. Andújar, "A proposal of energy management strategy on hybrid renewable system with hydrogen backup," in 2016 7th International Renewable Energy Congress (IREC), pp. 1-6, 2016.

[9] K.Y. Lau, M. Yousof, S. Arshad, M. Anwari and A. Yatim, "Performance analysis of hybrid photovoltaic/diesel energy system under Malaysian conditions," Energy, vol. 35, pp. 3245-3255, 2010.

[10] H. Kim, S. Baek, E. Park and H.J. Chang, "Optimal green energy management in Jeju, South Korea-On-grid and off-grid electrification," Renewable Energy, vol. 69, pp. 123-133, 2014.

[11] M.S. Behzadi and M. Niasati, "Comparative performance analysis of a hybrid PV/FC/battery stand-alone system using different power management strategies and sizing approaches," Int J Hydrogen Energy, vol. 40, pp. 538-548, 2015.

[12] A. Maheri, "Effect of dispatch strategy on the performance of hybrid wind-PV battery-diesel-fuel cell systems," Journal of Thermal Engineering, vol. 4, pp. 820-825, 2016.

[13] A. Maheri, "A critical evaluation of deterministic methods in size optimisation of reliable and cost effective standalone hybrid renewable energy systems," Reliab.Eng.Syst.Saf., vol. 130, pp. 159-174, 2014.

[14] P. Moseley, "Fuel Cell Systems Explained-James Larminie, Andrew Dicks (Eds.), Wiley, Chichester, Weinhein, New York, Brisbane, Singapore and Toronto, ISBN 0-471-49026-1," J.Power Sources, vol. 93, pp. 285-285, 2001

[15] O. Schmidt, A. Gambhir, I. Staffell, A. Hawkes, J. Nelson and S. Few, "Future cost and performance of water electrolysis: An expert elicitation study," Int J Hydrogen Energy, vol. 42, pp. 30470-30492, 2017.

[16] R. Dufo-Lopez and J.L. Bernal-Agustín, "Multi-objective design of PV-wind-diesel-hydrogen-battery systems," Renewable Energy, vol. 33, pp. 2559-2572, 2008. 